INPLASY

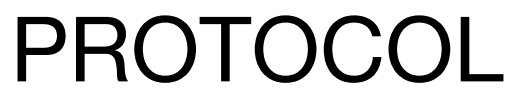

To cite: Zhang et al. Prognostic role of neutrophil-ToLymphocyte ratio in oropharyngeal cancer: A systematic review and metaanalysis including 6,987 patients. Inplasy protocol 202090032. doi: 10.37766/inplasy2020.9.0032

Received: 07 September 2020

Published: 07 September 2020

Corresponding author: Chuan Qin

qinchuan@pumc.edu.cn

Author Affiliation: Institute of laboratory animal sciences, CAMS\&PUMC

Support: None.

Review Stage at time of this submission: Data extraction.

Conflicts of interest:

The authors declare no conflict of interest.

\section{Prognostic role of neutrophil-To- Lymphocyte ratio in oropharyngeal cancer: A systematic review and meta-analysis including 6,987 patients}

Zhang, DY1; Zhang, L2; Zhu, XC3; Li, JW4; Qin, C5.

Review question / Objective: P: oropharyngeal cancer patients; I: High NLR group; C: Low NLR group; O: Overall survival; S: Cohort study.

Condition being studied: We divided oropharyngeal cancer patients into high NLR value group and low NLR value group to find the prognosis role of NLR.

Information sources: PubMed, Embase, Cochrane library and Web of Science databases.

INPLASY registration number: This protocol was registered with the International Platform of Registered Systematic Review and Meta-Analysis Protocols (INPLASY) on 07 September 2020 and was last updated on 07 September 2020 (registration number INPLASY202090032).

\section{INTRODUCTION}

Review question / Objective: P: oropharyngeal cancer patients; I: High NLR group; C: Low NLR group; O: Overall survival; S: Cohort study.

Condition being studied: We divided oropharyngeal cancer patients into high
NLR value group and low NLR value group to find the prognosis role of NLR.

\section{METHODS}

Participant or population: Patients with oropharyngeal cancer(confimed by histopatholopy) and no metastases from other sites to the cancer. 
Intervention: High NLR value.

Comparator: Low NLR value.

Study designs to be included: Both retrospective study and prospective study can be included.

Eligibility criteria: (i) Studied patients with oropharynx tumor were pathological examination confirmed; (ii) NLR was measured by serum based methods; (iii) Correlation of NLR with overall survival (OS) (iv) The study type of article should be cohort study Exclusion criteria were as follows: (i)Abstracts, letters, case reports, reviews or nonclinical studies; (ii) Studies were not written in English; (iii) Studies with insufficient data for estimating hazard ratio (HR) and 95\% confidence interval (Cl); (iv) Studies had duplicate data or repeat analysis. (v) Full text cannot be obtained.

Information sources: PubMed, Embase, Cochrane library and Web of Science databases.

Main outcome(s): Hazard ratio (HR), 95\% confidence Interval $(\mathrm{Cl})$, overall survival (OS).

Quality assessment / Risk of bias analysis: The Newcastle-Ottawa Scale (NOS) was used to assess each of the included studies quality.Begg's funnel plot and the Egger's linear regression test were performed to evaluate publication bias.

Strategy of data synthesis: We directly obtained $\mathrm{HR}$ and $95 \% \mathrm{Cl}$ from each literature or estimated these data according to the data obtained from survival curves with Engauge Digitizer and program based on the method of Jayne $F$ Tierney. HR $>1$ indicated a worse prognosis for oropharynx tumor patients with high expression of NLR. Cochran's $Q$ test and Higgins I-squared statistic were undertaken to assess the heterogeneity of the included trials. We use random effects (DerSimonian-Laird method) models were used to calculate the pooled HRs and $95 \% \mathrm{Cls}$ because of the data included had heterogeneity. In subgroup analysis to find the heterogeneity, Pheterogeneity $50 \%$ suggested significant heterogeneity in the literature and both random-effect model and fix-effect model was used. Subgroup analysis was conducted to explore and explain the diversity (heterogeneity) among the results of different studies. Publication bias was assessed by Begg's and Egger's funnel plot. All p-values were two-sided. A $p<0.05$ was considered statistical significant. All the statistical analyses were performed using Stata statistical software version 12.0.

Subgroup analysis: Subgroup analysis was conducted to explore and explain the diversity (heterogeneity) among the results of different studies.We divided the study into several different subgroups based on the sample size, nation, cut-off value of NLR and so on.

Sensibility analysis: We will use STATA for sensitivity analysis.

Country(ies) involved: China.

Keywords: oropharyngeal cancer, neutrophil-to-lymphocyte ratio, overall survival.

Contributions of each author:

Author 1 - Dongyuan Zhang.

Author 2 - Ling Zhang.

Author 3 - Xuchao Zhu.

Author 4 - Jingwen Li.

Author 5 - Chuan Qin. 